\title{
IMPROVED HYBRID DIFFERENTIAL EVOLUTION BY SELF ADAPTIVE RECOMBINATION OPERATOR TO SOLVE UNIT COMMITMENT PROBLEM OF THERMAL GENERATORS
}

\author{
M. Ramu ${ }^{1}$, L. Ravi Srinivas ${ }^{2}$, S. Tara Kalyani ${ }^{3}$
}

Abstract- This paper proposes a novel Improved Hybrid Differential Evolution (IHDE) combined with Lagrangian relaxation (LR) technique to solve the unit commitment problem (UCP) of Thermal generators. Improved Hybrid differential evolution (IHDE) is a simple population based stochastic function method. This method retains the properties of HDE and borrows the concept of Self Adaptive Recombination (SAR) operator. SAR operator converges toward local optimum solutions. In this paper IHDE algorithm has been implemented to tune the Lagrangian multipliers more effectively. The UCP is solved by the LR method. The proposed approach is tested on 10 units to 100 unit's system with 24 hours' demand horizon and the results attained are compared with other methods to verify its effectiveness.

Keywords- Unit Commitment (UC), Lagrangian Relaxation (LR), Hybrid Differential Evolution (HDE), Improved Hybrid Differential Evolution (IHDE)

\section{INTRODUCTION}

The objective of Unit Commitment (UC) is to schedule most cost-effective blend of generating units to meet estimated load and reserve necessities. The UC is made for a time perspective of one day to one week and determines which generators should be operating for the duration in hours. This commitment schedule takes into account of the inter-temporal parameters of each generator (minimum up time, minimum down time etc.) but does not specify production levels, which are determined a few minutes before delivery. The determination of these levels is known as economic dispatch and it is "the least-cost usage of the committed resources during a single period to meet up the demand" [1]. Unit commitment is a non-linear, non-convex large scale problem. It is difficult to find the optimal solution for a large-scale problem $[2,3]$. Several optimization methods are at present used and many more are being researched. Literature review identified a few of these methodologies i.e. Priority list method [4], dynamic programming [5], Lagrangian relaxation [6]. Due to the high complexity and high nonlinearity of the UC problem, the solution obtained from the above mentioned methods are encountered with convergence problems which results in poor solution. Recently many non-classical methods have been developed which comprise of, Artificial Neural Networks [7], genetic algorithm[8], simulated annealing[9], tabu-search method[10], fuzzy logic algorithm [11], cone programming[12], particle swarm optimization[13], differential evolution [14-16], Hybrid differential evolution [17,18] and its modifications[1921], evolutionary programming [22], combination of fuzzy and simulation annealing [23], improved quantum evolutionary algorithm [24], Hybrid differential evolution[25] to solve various engineering problems. This paper presents an efficient algorithm that is Improved Hybrid Differential Evolution (IHDE) incorporating Lagrangian relaxation method is proposed to solve the UC problem. The UC problem is solved by LR method where as Lagrangian multipliers are initiated and updated by IHDE algorithm.

\section{PROBLEM FORMULATIONS}

Objective of a unit commitment problem is to minimize the production cost over the scheduled time horizon (24hours) under the generator operational and spinning reserve constraints. Objective function of UC to be minimized is

$$
F\left(S_{i}^{t}, V_{i, t}\right)=\sum_{t=1}^{T} \sum_{i=1}^{N} F_{i}\left(S_{i}^{t}\right)+S T_{i, t}\left(1-V_{i, t-1}\right) V_{i, t}
$$

Subject to following constraints

$$
\begin{aligned}
& 2.1 \text { Power balance constraint- } \\
& \sum_{i=1}^{N} S_{i}^{t} V_{i, t}=S_{D}^{t}
\end{aligned}
$$

\footnotetext{
${ }^{1}$ Department of Electrical and Electronics Engineering, GITAM University, Visakhapatnam, India

${ }^{2}$ Department of Electrical and Electronics Engineering, Gudlavalleru Engineering College, Gudlavalleru, India

${ }^{3}$ Department of Electrical and Electronics Engineering, JNTU University, Hyderabad, India
} 
2.2 Spinning reserve constraint -

$\sum_{i=1}^{N} S_{i, \max } V_{i, t} \geq S_{D}^{t}+R^{t}$

2.3 Generator limit constraints -

$S_{i, \min } V_{i, t} \leq S_{i}^{t} \leq S_{i, \max } V_{i, t}, i=1, \ldots, N$

2.4 Minimum up and down time constraints-

$V_{i, t}= \begin{cases}1, & \text { if } T_{i, o n}<T_{i, u p} \\ 0, & \text { if } T_{i, o f f}<T_{i, d o w n} \\ 0 \text { orl }, & \text { otherwise }\end{cases}$

\subsection{Startup cost-}

$S T_{i, t}=\left\{\begin{array}{l}H S C \text { if } T_{i, \text { down }} \leq T_{i, \text { off }} \leq T_{i, \text { cold }}+T_{i, \text { down }} \\ C S C \text { if } T_{i, \text { off }}>T_{i, \text { cold }}+T_{i, \text { down }}\end{array}\right.$

\section{LAGRANGIAN RELAXATION}

The LR procedure resolves the UC problem by relaxing or temporarily ignoring the coupling constraints and solving the problem as if they did not exist. This is done through the dual optimization. [6]

$L(S, V, \lambda, \mu)=F\left(S_{i}^{t}, V_{i, t}\right)+\sum_{i=1}^{T} \lambda^{t}\left(S_{D}{ }^{t}-\sum_{i=1}^{N} S_{i}^{t} V_{i, t}\right)+\sum_{t=1}^{T} \mu^{t}\left(S_{D}{ }^{t}+R^{t}-\sum_{i=1}^{N} S_{i, \max } y_{i . t}\right)$

With respect to nonnegative $\lambda \mathrm{t}$ and $\mu \mathrm{t}$, whereas minimizing it with respect to other control variables in problem, that is

$q^{*}\left(\lambda_{s} \mu\right)=\operatorname{Max}_{\lambda} \mu^{\mathrm{t}} \mu^{\mathrm{t}} q\left(\lambda_{s} \mu\right)$

Where

$q(\lambda, \mu)=\operatorname{Min} S_{i}^{t} w_{i, t} L(S, S, \lambda, \mu)$

Equations (2) \& (3) are coupling constraints across the thermal units.

Lagrangian function is rewritten as

$$
\left.L=\sum_{i=1}^{N} \sum_{t=1}^{F}\left\{F\left(S_{i}^{t}\right)+S T_{i, t}\left(1-V_{i, t-1}\right)\right] V_{i, t}-\lambda t S_{i}^{t} V_{i, t}-\mu S_{i, \max } V_{i, t}\right\}+\sum_{t=1}^{T}\left(\lambda S_{D}{ }^{t}+\mu\left(S_{D}{ }^{t}+R^{t}\right)\right.
$$

The term

$$
\sum_{t=1}^{T}\left\{F\left(S_{i}^{t}\right)+S T_{i, t}\left(1-V_{i, t-1}\right) V_{i, t}-\chi S_{i}^{t} V_{i, t}-\mu S_{i, \max } V_{i, t}\right\}
$$

can be minimized separately by each thermal generating unit, when the coupling constraints are temporarily ignored. Then, the minimum of the Lagrangian function is solved for each generating unit over the time horizon, that is

$$
\begin{aligned}
& \operatorname{Min} S, V_{i, t} L(S, V, \lambda, \mu)= \\
& \sum_{t=1}^{N} \min \sum_{t=1}^{T}\left\{F\left(S_{i}^{t}\right)+S T_{i, t}\left(1-V_{i, t-1}\right) V_{i, t}\right\}-\lambda t S_{i}^{t} V_{i, t}-\mu t S_{i, \max } V_{i, t}
\end{aligned}
$$

Subjected to $V_{\mathrm{i}, t} S_{i \min } \leq S_{\mathrm{i}}^{\mathrm{t}} \leq V_{\mathrm{i}, \mathrm{t}} S_{\text {imax }}$

for $\mathrm{t}=1, \ldots, \mathrm{T}$ and the constraints in equation (5)

On/Off decision criterion:

In the Lagrangian relaxation method, the dual solution is obtained using dynamic programming for each unit separately. This can be visualized in fig. 1 showing the only two possible states for unit i (i.e, $U_{i, t}=0$ or 1 ). At $U_{i, t}=0$ state, the value of the function to the minimized is unimportant (i.e., it equals zero), at the state where $U_{i, t}=1$, the function to be minimized is $\left[F_{i}\left(P_{i}^{t}\right)-\lambda^{t} P_{i}^{t}\right]$ the startup cost and the term $\mu^{t} P_{i, \max }$ are dropped here since the minimization is with respect to $P_{i}^{t}$

To find the dual power, the term $\min \left[E_{i}\left(P_{i}^{t}\right)-\lambda^{t} P_{i}^{t}\right]$ will be minimized by the optimality condition 


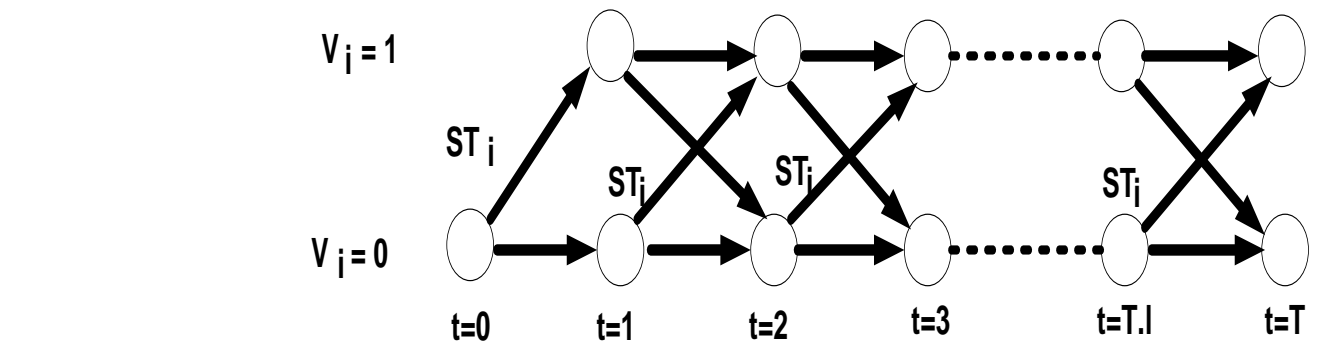

$$
\frac{d}{d P_{i}}\left[F_{i}\left(P_{i}^{t}\right)-\lambda^{t} P_{i}^{t}\right]=0
$$

Figure 1. Two-state dynamic programming

The solution to this equation is

$$
\frac{d F_{i}\left(S_{i}^{t, d u a l}\right)}{d S}=\lambda^{t}
$$

The dual power is obtained

$S_{i}^{t, d u a l}=\frac{\lambda^{t}-b_{i}}{2 C_{i}}$

Three cases to check $P_{i}^{t_{1} \text { opt }}$ against its limits

A. If $S_{i}^{\text {td dual }}<S$, Then $S_{i}^{t}=S_{\text {imin }}$

B. If $S_{\text {imin }} \leq S_{i}^{\text {tidual }} \leq S_{\text {imax }}$ ?

Then $S_{i}^{t}=S_{i}^{t, d u a l}$

C. If $S_{i}^{t d d u a l}>P_{\text {imax } x}$ Then $S_{i}^{t}=S_{\text {imax }}$

Dynamic programming is employed to decide the optimal schedule of each unit over the scheduled horizon. More specifically, for each state in each hour, the on/off decision making is needed to select the lower cost by comparing the combination of the start-up cost and accumulated costs from two historical routes. The dual power calculated will be substituted in the new On/Off decision criterion.

$\left\lfloor F_{i}\left(S_{i}^{t}\right)+S T_{i, t}\left(1-V_{i, t-1}\right)\right\rfloor-\lambda^{t} S_{i}^{t}-\mu t S_{i}^{t}-\mu t S_{i, \max }^{t}$

$\mathrm{i}=1 \ldots \mathrm{NP}$

To minimize the above term in equation (15) at each hour, if $\left.\left[F_{i}\left(S_{i}^{t}\right)+S T_{i, t}\left(1-V_{i, t-1}\right)\right]-\lambda^{t} S_{i}^{t}-\mu^{t} S_{i, \max }^{t}\right\} \leq 0$, this unit will be committed, if it does not violate the minimum downtime constraint $\left(U_{i, t}=1\right)$.

\section{OVERVIEW OF HYBRID DIFFERENTIAL EVOLUTION [18, 21]}

Hybrid differential evolution (HDE) is a simple population based stochastic method that comprises an addition from the unique algorithm of DE as initiated by Storn and Price. The original algorithm of DE was used to solve the unconstrained nonlinear optimization problems. Chiou and Wang have extended the original DE to solve optimal control problem.

Basic operations in HDE are

- Initialization

- Mutation

- Crossover

- Selection and evaluation

- Migration

\section{IMPROVED HYBRID DIFFERENTIAL EVOLUTION METHOD [25]}

IHDE method retains the basic operations of HDE and borrows concept of self-adaptive recombination operator invoked in mutation. In order to investigate results due to self-adaptive recombination operator, the algorithm is summarized as follows. 


\subsection{Initialization -}

The initial populations are chosen randomly and would attempt to cover the whole parameter space without fail. Uniform likelihood allotment for all random variables is assumed, that is

$\mathrm{Xi} 0=\mathrm{X} \min +\rho \mathrm{i}(\mathrm{X} \max -\mathrm{X} \min )$

Where $\rho \mathrm{i} \epsilon[0,1]$ is a random number. The initial development makes NP individuals of Xi0 randomly.

\subsection{Mutation-}

A mutant vector is generated based on the nearby individual $\mathrm{XiG}$ as follows

$\mathrm{YiG}+1=\mathrm{XiG}+\mathrm{F}((\mathrm{Xr} 1 \mathrm{G}-\mathrm{Xr} 2 \mathrm{G})+(\mathrm{Xr} 3 \mathrm{G}-\mathrm{Xr} 4 \mathrm{G}))$

According to mutation constant $F$ was chosen as $\mathrm{F}^{\in}[0,1.2]$, and the upper limit of 1.2 for $\mathrm{F}$ was determined empirically;

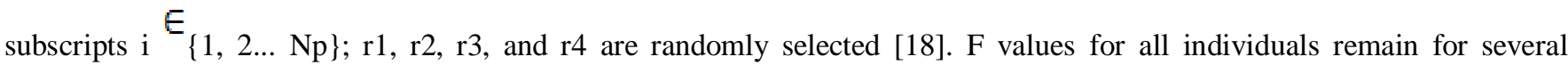
generations and then a new set of $\mathrm{F}$ value is generated under the same normal distribution.

A. $\quad$ Create a new individual via recombination and repeat it for 5 times

B. Copy new individual for each block in the individual

C. Decode mutation rate of block $\mu$ block for each gene $\mathrm{i}$ in the block $\mu \mathrm{i}=\mu \mathrm{i}+\mathrm{N}(0, \mu$ block $)$

D. Decode fresh mutation rate of block $\mu$ block for every gene $\mathrm{i}$ in the block mutate with probability $\mu^{\prime}$ block (selfadaptive recombination operator)

E. Evaluate this individual

Select the finest individual from the offspring and replace the oldest member of population.

\subsection{Crossover-}

In order to boost the diversity among the individuals of the next generation, a perturbed individual, YiG+1, and a present individual, XiG, are chosen by a binomial distribution to progress the crossover operation to generate an offspring. Each gene of the ith individual is reproduced from the mutant vector $\mathrm{YiG}+1=(\mathrm{Y} 1 \mathrm{iG}+1, \mathrm{Y} 2 \mathrm{iG}+1, \ldots \ldots . \mathrm{YkiG}+1)$ and the present individual $\mathrm{XiG}=(\mathrm{X} 1 \mathrm{iG}, \mathrm{X} 2 \mathrm{iG}, \ldots \ldots . . . . \mathrm{XkiG}+1)$.

That is

$$
S_{i h i}^{G+1}= \begin{cases}X_{i h i}^{G+1}, & \text { if arandomumbe } C_{r} \\ S_{h i}^{G+1 Y}, & \text { otherwist }\end{cases}
$$

Where $\mathrm{i}=1,2, . . N_{\mathrm{p}} ; \mathrm{h}=1, \ldots, n_{\mathcal{C}} ; n_{C}$ is the dimension of decision parameters; and the crossover constant is set to be $\mathrm{Cr} \epsilon[0,1]$.

\subsection{Estimation and Selection}

The parent is replaced by its progeny if the fitness of the progeny is better than that of the parent. The parent is retained in the next generation if the fitness of the progeny is poorer than that of the parent. Two forms are presented as follows

$\mathrm{XiG}+1=\arg \min \{\mathrm{F}(\mathrm{XiG}), \mathrm{F}(\mathrm{YiG}+1)\}$

$\mathrm{XbG}+1=\arg \min \{\mathrm{F}(\mathrm{XiG}+1)\}$

Where arg min means the argument of the minimum, and $\mathrm{XbG}+1$ is the best individual.

\subsection{Migration If Necessary -}

In order to enhance the examination to the search spaces successfully, reduce the choice weight to a small population, a migration operation is started to regenerate a new diverse population of individuals. The new populations are yielded based on the best individual $\mathrm{XbG}+1$. The hth gene of the ith individual is as follows

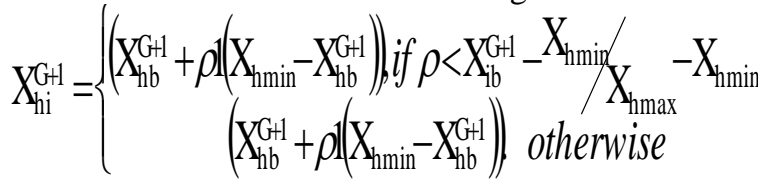

Where $\rho 1, \rho 2$ are randomly generated numbers uniformly distributed in the range of $[0,1] ; \mathrm{i}=1, \ldots . \mathrm{Np} ; \mathrm{h}=1, \ldots ., n_{G}$. Migration in $\mathrm{HDE}$ is carryout only if a measure fails to match the desired tolerance of population diversity. This measure is defined as follows 


$$
\begin{aligned}
& \rho=\sum_{\substack{i=1 \\
i \neq b}}^{N p} \sum_{j=1}^{n C} \frac{X_{i j}}{n_{c}}(N p-1)<\varepsilon 1 \\
& \text { Where } X_{j i}= \begin{cases}1_{v} & \text { if }\left|\frac{x_{j i}^{C+1}-x_{j b}^{C+1}}{x_{j b}^{C+1}}\right|>\varepsilon_{2} \\
0, & \text { otherwise }\end{cases}
\end{aligned}
$$

Parameters $\varepsilon 1 \in[0,1]$ and $\varepsilon 2 \in[0,1]$ respectively express the desired tolerance for the population diversity and the gene diversity with respect to the best individual. Here $X_{\mathrm{ij}}$ is defined as an index of the gene diversity. A value of zero for $X_{\mathrm{ij}}$ denotes that the jth gene of the ith individual is very close to the jth gene of the best individual. From Eqs. (22) and (23), it can be seen that the value of $\rho$ is in the range of $[0,1]$. If $\rho$ is smaller than $\varepsilon 1$, then the HDE performs the migration to generate a new population to escape the local point; otherwise, the HDE breaks off the migration and keeps an ordinary search direction. 6 Repeat steps 5.2 to 5.5 until the maximum iteration quantity or the desired fitness is reached.

\section{IMPLEMENTATION OF PROPOSED IHDE METHOD}

The cost of power generation is calculated using lambda iteration based on the status of each power generating unit. For each hour depending on whether the start-up is cold start or a hot start, the appropriate cost is added to the total cost. A penalty term is used if the hourly power demands plus a specific amount of reserve is not meant or if and constraints are violated. For implementing the proposed solution, the population is set to be 50 and iterations are 250 . The mutation constant and crossover constant taken are 0.5 and 0.8 respectively. Parameter values are determined using the best settings formed as a result of a series of 10 runs. The main computational procedure of HDE is given in step 5.1 to step 5.6.

In step 5.2 self-adaptive recombination operators is added to mutation results in IHDE, it enhances the mutation rate. The proposed method mainly involves the application of IHDE algorithm to the UC problem. IHDE have been tested initially to the 10 generator system and later extended to 100 generator systems. 10 runs were carried out for each set from 10 to 100 generators for both HDE and IHDE methods. The best of the runs is considered as optimum solution. Results are compared with the existing methods for verifying robustness of proposed methods.

The fitness function to be minimized as follows

$$
f=\sum_{t=1}^{T}\left\{\sum_{i=1}^{N}\left[F_{i}(s)+S T_{i_{\mu} t}\left(1-V_{i_{\mu} t-1}\right)\right] V_{i_{\mu} t}+k_{g} \sum\left(s_{p}-s_{\text {plim }}\right)^{2}\right\}+k_{u} \sum\left(T_{U}-T_{\text {ulim }}\right)^{2}+k_{d} \Sigma\left(T_{D}-T_{d l i m}\right)^{2}
$$

The computation finds better unit commitment with least cost so that the value of the objective function is successfully reduced.

\section{RESULTS}

The proposed algorithm for solving unit commitment problem was programmed in MATLAB of version 2010a environment with Pentium dual core, $3.2 \mathrm{GHz}$ computer with 3 GB RAM. The test system consisting of 10 to 100 power generating units and a time horizon of 24 hours is taken from [8].. Unit commitment schedule of IHDE given in Table 1, Fig. 2 shows the convergence of total cost for 100-unit system of IHDE and Fig. 3 shows the fitness of UC solution, Simulation results of the proposed IHDE method is given in Table 2, Comparison of results of proposed method with other methods is given in Table 3.

Table -1 Unit commitment schedule of 100 generator system

\begin{tabular}{|l|l|}
\hline Hour & Units On/Off Schedule \\
\hline & \\
\hline
\end{tabular}


Improved Hybrid Differential Evolution By Self Adaptive Recombination Operator To Solve Unit Commitment Problem Of

\begin{tabular}{|c|c|}
\hline & 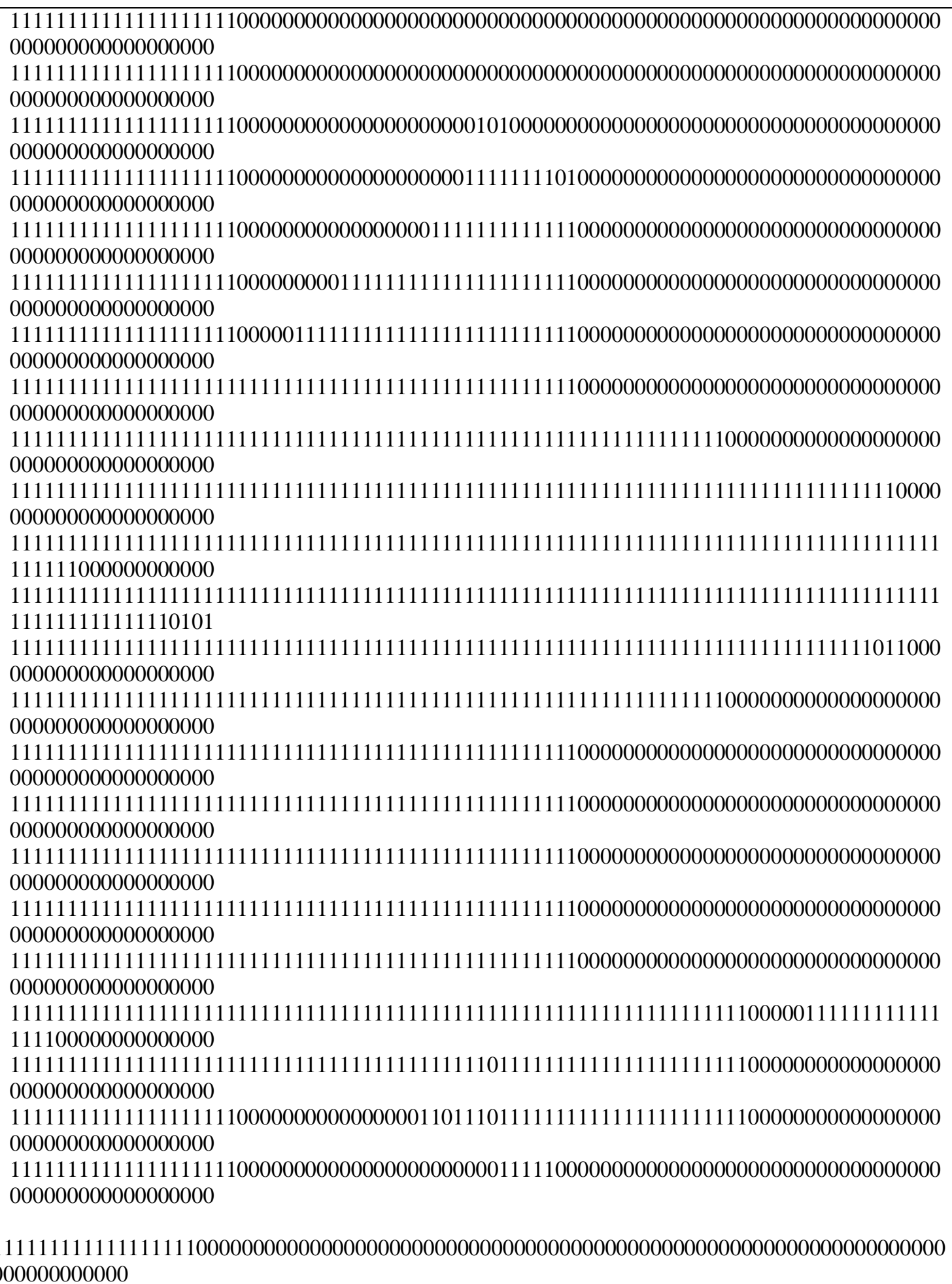 \\
\hline
\end{tabular}

Table -2 Simulation results of the proposed IHDE method

\begin{tabular}{|l|l|l|l|}
\hline $\begin{array}{l}\text { No. of } \\
\text { Units }\end{array}$ & $\begin{array}{l}\text { Best cost } \\
(\$)\end{array}$ & $\begin{array}{l}\text { Average cost } \\
(\$)\end{array}$ & $\begin{array}{l}\text { Worst cost } \\
(\$)\end{array}$ \\
\hline 10 & 563,966 & 565,820 & 570,409 \\
\hline 20 & $1,123,297$ & $1,126,253$ & $1,129,129$ \\
\hline 40 & $2,243,363$ & $2,250,621$ & $2,254,124$ \\
\hline
\end{tabular}




\begin{tabular}{|l|l|l|l|}
\hline 60 & $3,369,534$ & $3,372,350$ & $3,378,408$ \\
\hline 80 & $4,486,242$ & $4,492,731$ & $4,505,282$ \\
\hline 100 & $5,604,951$ & $5,621,910$ & $5,630,959$ \\
\hline
\end{tabular}

Table -3 Comparison of various methods

\begin{tabular}{|l|l|l|l|l|l|l|l|}
\hline \multirow{2}{*}{$\begin{array}{l}\text { No of } \\
\text { generators }\end{array}$} & \multicolumn{1}{l}{ Total cost (\$) } \\
\cline { 2 - 9 } & LR [6] & GA [6] & EP [6] & Fuzzy SADP [11] & IQEA [24] & HDE & IHDE \\
\hline 10 & 565,825 & 565,825 & 564,551 & 563,978 & 563,977 & 563,977 & 563,966 \\
\hline 20 & $1,130,660$ & $1,126,243$ & $1,125,494$ & $1,123,390$ & $1,123,890$ & $1,123,921$ & $1,123,555$ \\
\hline 40 & $2,258,503$ & $2,251,911$ & $2,249,093$ & $2,244,334$ & $2,245,151$ & $2,246,821$ & $2,246,803$ \\
\hline 60 & $3,394,066$ & $3,376,625$ & $3,371,611$ & $3,366,975$ & $3,365,003$ & $3,370,001$ & $3,369,534$ \\
\hline 80 & $4,526,022$ & $4,504,933$ & $4,498,479$ & $4,490,844$ & $4,486,963$ & $4,494,279$ & $4,486,242$ \\
\hline 100 & $5,657,277$ & $5,627,437$ & $5,623,885$ & $5,610,217$ & $5,606,022$ & $5,605,561$ & $5,604,622$ \\
\hline
\end{tabular}

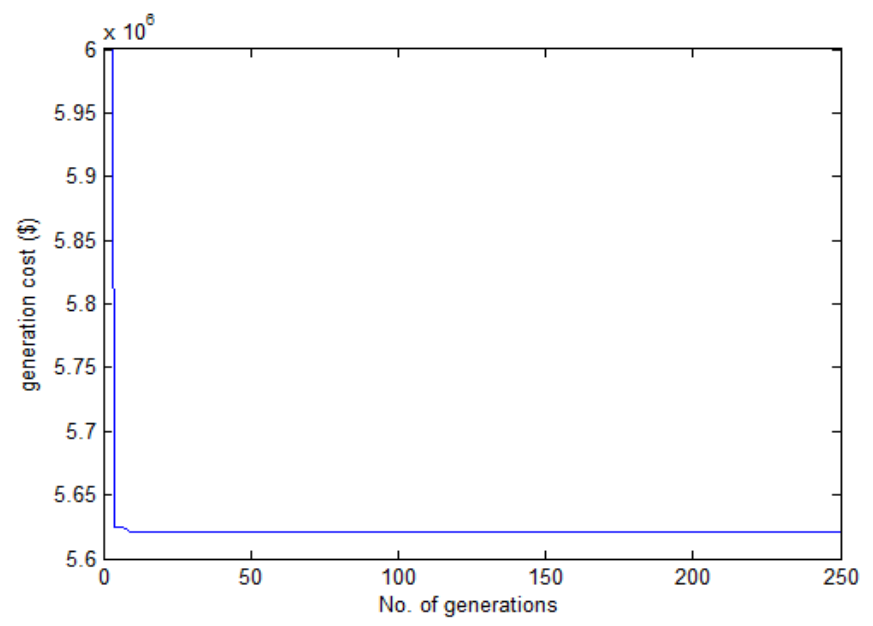

Figure 2. Convergence of total cost of 100-unit system with IHDE algorithm

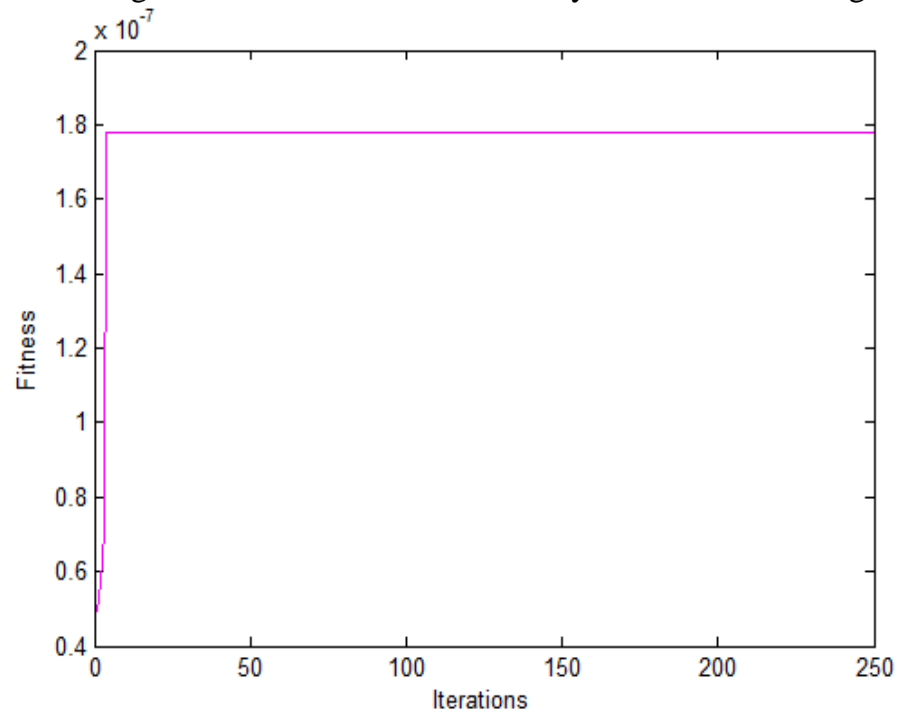

Figure 3. Convergence of fitness of 100-unit System with IHDE algorithm

\section{CONCLUSIONS}

This paper proposes new method using Improved Hybrid Differential Evolution optimization algorithms for solving the problem of UC. The proposed algorithms' effectiveness is tested on 10 to 100 generator systems. The total production costs by 
IHDE over the schedule of $24 \mathrm{hr}$ horizon are reduced when compared with other optimization methods reported in the literature. Results demonstrated that IHDE is a robust method to solve the UC problem.

\section{REFERENCES:}

[1] J. Wood and B. F. Wollenberg, "Power Generation, Operation \& Control”, 2nd ed. New York: Wiley, 2007.

[2] Dimitris Bertsimas, Eugene Litvinov, Xu Andy Sun, Jinye Zhaoand Tongxin Zheng, “Adaptive Robust Optimization for the Security Constrained Unit Commitment Problem", IEEE Trans. Power Syst., vol. 28, no. 1, Feb. 2013.

[3] A. Bhardwaj et al., "Unit Commitment in Electrical Power System - a Literature Review ", IEEE International Power Engineering and Optimization Conference, Melaka, Malaysia, 6-7 June 2012

[4] Yang Tingfang, T. O. Ting, "Methodological Priority List for Unit Commitment Problem”, IEEE computer soci ety, pp.176-179, 2008.

[5] Snyder Jr. W. L., Powell Jr. H. D., and Rayburn J. C., "Dynamic programming approach to unit commitment", IEEE Trans. on Power Apparatus and Systems, Vol. PAS-2, pp. 339-350, May 1987.

[6] Ongsakul,W., Petcharaks, "Unit commitment by enhanced adaptive lagrangian relaxation", IEEE Trans. Power Syst, $19(1), 620-628,2004$.

[7] Musoke H. Sendaula and Saroj K Biswas, Ahmed Eltom and Cliff Parten, Wilson kazibwe, "Application of Artificial Neural Networks to Unit Commitment.", IEEE Trans. Power Syst., pp.256-260, 1991.

[8] S. A. Kazarlis, A. G. Bakirtzis and V. Petridis, "A genetic algorithm solution to the unit commitment problem", IEEE Trans. Power Syst., vol. 11, pp. 83-92, Feb. 1996.

[9] Dimitris N. Simopoulos, Stavroula D. Kavatza, D.Vournas, "Unit Commitment by an Enhanced Simulated Annealing Algorithm", PSCE, pp.193-201, 2006.

[10] C. Christo, Asir Rajan and M. R. Mohan, "An Evolutionary Programming-Based Tabu Search Method for Solving the Unit Commitment Problem", IEEE Trans. Power Syst., pp-557-585, vol.19, Feb. 2004.

[11] D. P. Kadam, P. M. Sonwane, V. P. Dhote, B. E. Kushare, "Fuzzy Logic Algorithm for Unit Commitment Problem", International conference on control, automation, communication and energy conservation, pp 1-4, 2009.

[12] Xiaohui Yuan Hao, Tian Shuangquan Zhang Bin Ji, Yanhong Hou, "Second-order cone programming for solving unit commitment strategy of thermal generators", Energy Conversion and Management, vol.76, pp.20-25, 2013.

[13] Balci H.H., Valenzuela J.F. "Scheduling electric power generators using particle swarm optimization combined with the Lagrangian relaxation method", Int.J. Appl. Math. Comput. Sc, 14 (3), pp. 411- 421, 2004.

[14] A. K. Qin, V. L. Huang and P. N. Suganthan, "Differential evolution algorithm with strategy adaptation for global numerical optimization", IEEE Transactions on Evolutionary Computation, vol. 13, no. 2, pp. 398-417, 2009.

[15] Yun-Won Jeong, Woo-Nam Lee, Hyun-Houng kim, Jong-Bae park and Joong-Rin shin”, Thermal Unit commitment using Binary Differential Evolution", Journal of Electrical Engineering \& Technology, vol 4 no3, pp 323-329, 2009.

[16] Price KV, Storn R, Lampinen J. Berlin "Differential evolution a practical approach to global optimization", Springer-Verlag; 2005.

[17] J. P. Chiou and F. S. Wang, "Hybrid method of evolutionary algorithms for static and dynamic optimization problems with application to fed-batch fermentation process", Comput. Chem. Eng., vol. 23, pp. 1277-1291, 1999.

[18] "Estimation of monod parameters by hybrid differential evolution," Bioprocess and Biosyst. Eng., pp. 109-113, 2001.

[19] Kit Po Wong and Zhao Yang Dong, "Differential Evolution, an Alternative Approach to Evolutionary Algorithm”, 1-59975-028-7/05, 2005.

[20] K.V. Price, "Differential evolution vs functions of the 2nd ICEC", in proceedings of the IEEE Conference on Evolutionary Computation, 1997, pp. 153157.

[21] Liang BAI, Junyan WANG, Youngheng JIAG, Dexian Huang "Improved Hybrid Differential Evolution Algorithm with feasibility rules for NLP/MINLP Engineering optimization problem”, Chinese journal of chemical engineering, Vol. 20, issue 6, Dec 2012, 1074-1080.

[22] K. A. Juste, H. Kita, E. Tanaka, and J. Hasegawa, “An evolutionary programming solution to the unit commitment problem”, IEEE Trans. Power Syst., vol. 14, pp. 1452-1459, Nov. 1999.

[23] S. Patra, S.K. Goswami and B. Goswami, "Fuzzy and simulated annealing based dynamic programming for the unit commitment problem", Expert Systems with Applications, vol.36, pp.5081-5086, 2009.

[24] Yun-Won Jeong, Jong-Bae Park, Joong-Rin Shin \& Kwang Y. Lee “A Thermal Unit Commitment Approach Using an Improved Quantum Evolutionary Algorithm”, Electric Power Components and Systems, 37:770-786, 2009.

[25] L. R. Srinivas, M. Ramu and S. T. Kalyani, "Implementation of hybrid differential evolution to solve unit commitment," 2015 IEEE 10th International Conference on Industrial and Information Systems (ICIIS), Peradeniya, 2015, pp. 440-445. 